thum entfaltet, nichts einwenden. Bildet jedoch eine die Primitivfibrillen umhüllende, flüssige Substanz einen wesent lichen Bestandtheil des Axencylinders, so stösst die Annahme eines directen Fortwachsens desselben auf grössere Schwierigkeiten, und es gewinnt die von mir vertretene Vorstellung an Wahrscheinlichkeit, dass sich zuerst eine Umwandlung desselben, eine Substitution durch eine protoplasmatische, wachsthumsfähige Masse, und zwar unter wesentlicher Betheiligung der sogen. „Zellen der Schwann'schen Scheide" vollzieht, und dass eine secundäre Differenzirung dieses Protoplasma zur Bildung der jungen Fasern führt. Die bisherigen Untersuchungen reichen leider nicht aus, hierüber definitiv zu entscheiden.

\title{
XXII.
}

\section{Ueber die Lage des Centrums der Macula lutea im menschlichen Gehirn.}

\author{
ron \\ Professor Dr. L. Laquear, \\ Director der Augenklinilk \\ und \\ Dr. Martin B. Schmidt, \\ Privatdocenten und I. Assistenten am Pathologiseben Institut \\ in Strassburg. \\ Hierzu Tafel XII und XIII.
}

Um die functionelle Bedeutung der einzelnen Theile des menschlichen Gehirns zu erkennen, besitzen wir kein sichereres Mittel, als das Studium der Ausfalls-Erscheinungen, welche nach Zerstörung umschriebener Hirnregionen eintreten. Dieselben werden jedoch nur dann sicher verwerthbar sein, wenn einer- 
Iirhow's Archin, Bd. (LIIII.

Tiaf XIII.

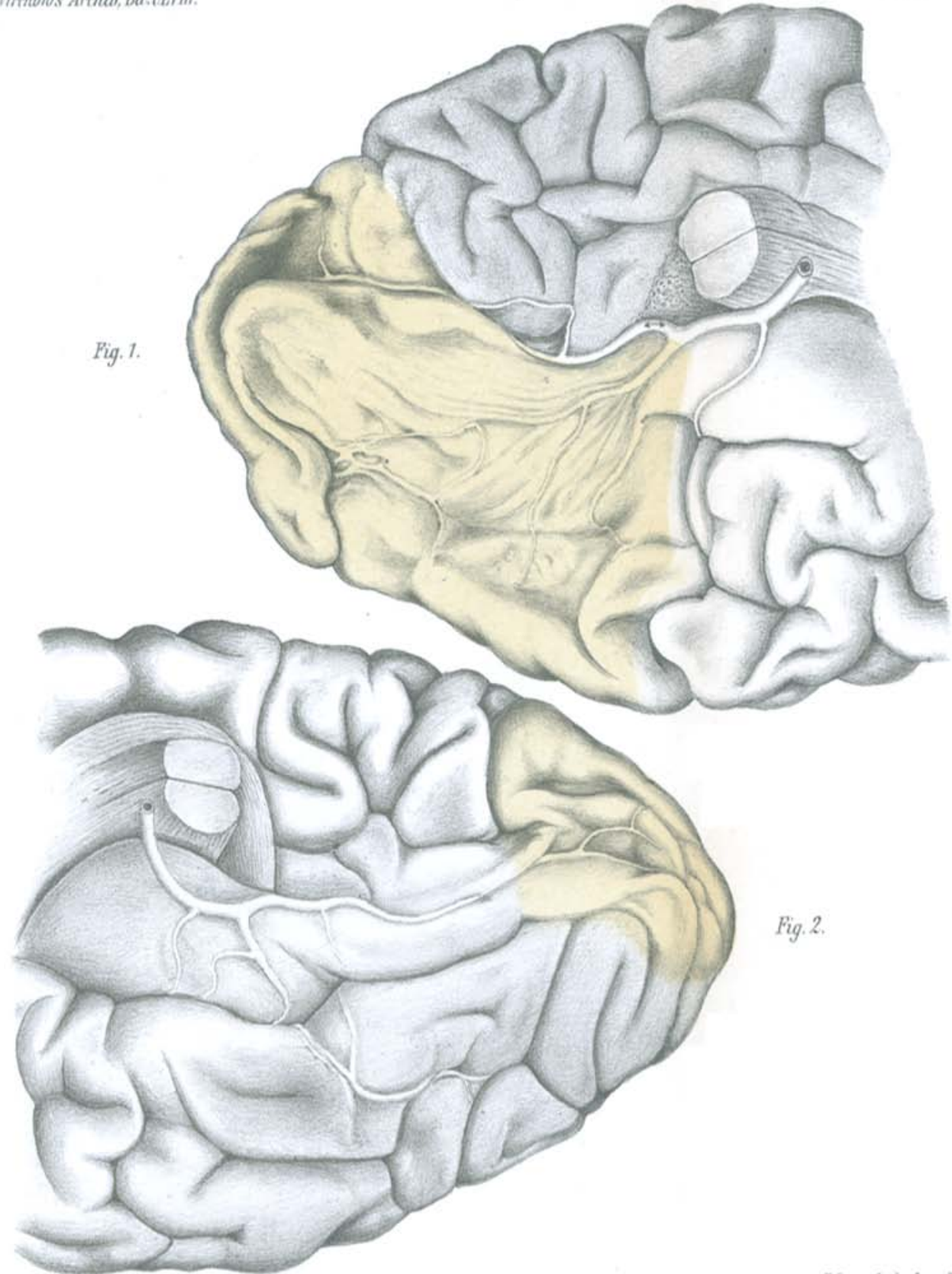

E Alocta qez.

E. Latus, Luth Snst. Berter 
L. 1.

nin?

feren

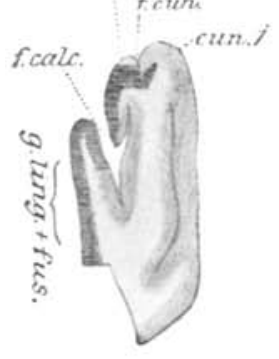

1. 2 .

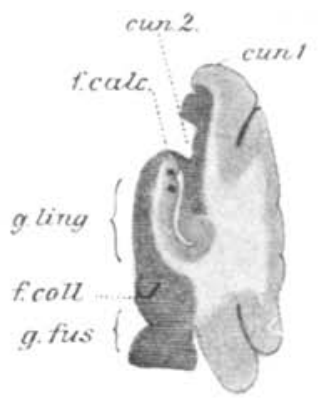

l. 3 .

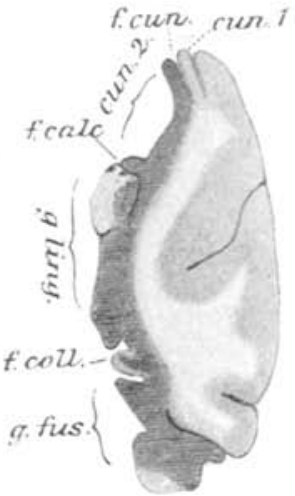

L. 6 .

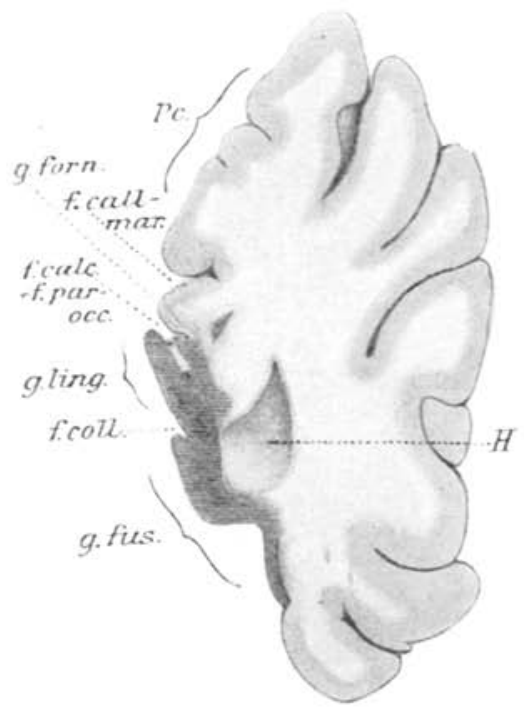

L. 4 .

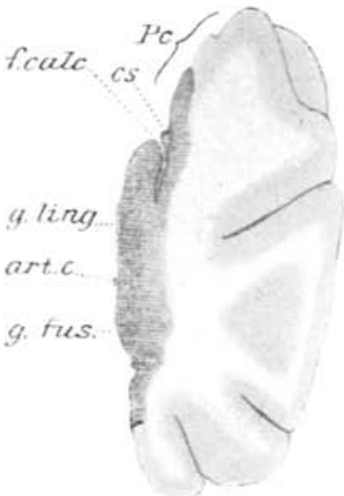

R. 3 .

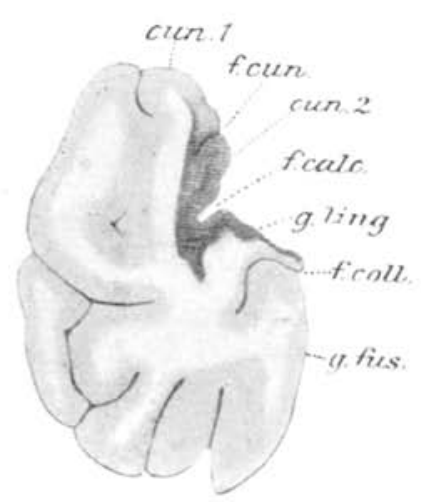

R. 2 .

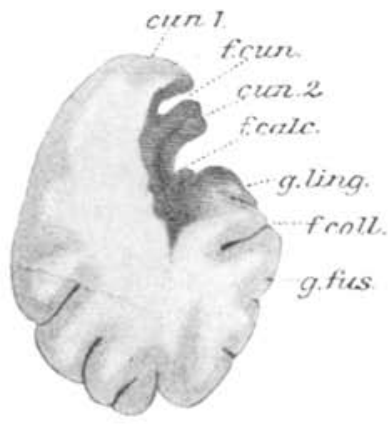


seits die klinisch zu constatirenden Störungen relativ einfach und gut erkennbar, andererseits die anatomischen Veränderungen des Gehirns deutlich ausgesprochen, scharf umschrieben und nicht mit Läsionen entfernter Gehirntheile complicirt sind. Nur in diesem Falle sind wir berechtigt, die erkrankte Partie des Gehirns als den Sitz der verloren gegangenen Function zu betrachten. Durch dieses Zusammenwirken klinischer Beobachtung und anatomischer Untersuchung sind wir zar Kenntniss des Sprachcentrums und anderer Centren der höheren seelischen Thätigkeiten des Menschen gelangt.

Einen lehrreichen Fall von eigenthümlicher Störung des Sehvermögens, bei welchem die anatomische Untersuchung des Gehirns ausgeführt werden konnte, sind wir in der Lage, hier mitzutheilen. $\mathrm{Er}$ wurde intra vitam von dem einen von uns beobachtet und in der vorjährigen Sitzung der ophthalmologischen Gesellschaft in Heidelberg mitgetheilt, in deren Jahresbericht die Krankengeschichte nebst dem makroskopischen Obductionsbefund veröffentlicht wurde. Sie ist in Kürze folgende:

Ein kräftiger, bis dahin völlig gesunder Mann erkrankte im Jahre 1884 (in seinem 48. Lebensjahre) an einer acuten Nephritis mit Hämaturie, die ihn 4 Wochen lang an's Bett fesselte; er erholte sich wieder, erkrankte aber im folgenden Jahre an einem ähnlichen Anfalle von acuter Nephritis. Seitdem litt er an Albuminurie, war aber dabei leistungsfähig.

Im Jahre 1896 kamen Besebwerden von Seiten des Herzens, Palpitationen und Beklemmungen, hinzu. Es wurde jetzt eine chronische Nephritis und allgemeine Arterio-Sklerose, auch Sklerose der Herzarterien diagnosticirt, ein Klappenfehler aber ausgeschlossen.

Am 15. Juni 1897 trat ganz plötzlich, beim Erwachen aus einem kurzen Mittagsschlaf, unter starken Stirnkopfschmerzen, aber ohne Pause des Bewusstseins und ohne Lähmungs-Erscheinungen, eine bedeutende Sehstörung ein. Am nächsten Tage fand sich bei der Untersuchung der Angen, welche in mässigem Grade hypermetropisch waren, rechts eine centrale Sehsclärfo von $\frac{3}{5}$, links von $\frac{3}{4}$ und eine typische Hemianopsie nach links. Im rechten Auge feblt die innere Hälfte des Gesichtsfeldes, die Trennungslinie verläuft genau vertical; im linken Auge fehlt die äussere Gesichtsfeld-Hälfte, hier ist die Trennungslinie ein wenig (etwa $2^{0}$ ) nach der kranken Seite ausgebogen. - Wilbrand's überschüssiges Gesichtsfeld. In den verloren gegangenen Hälften des Gesichtsfeldes hat der Patient absolut keine Empfindung - er sieht nicht Schwarz, sondern er sieht absolut Nichts - negatives Scotom. Die erhaltenen Hälften der Gesichtsfelder zeigen beide in der Peripherie eine leichte Einengung für Weiss, welehe nach oben hin bis $20^{\circ}$ beträgt. Der Farbensinn erweist sich beiderseits völlig normal. 
Objectiv war an den Augen und ibren Muskeln nichts Abnormes nachweisbar; die Pupillen reagirten gut auf Licht, und die Sehnerven zeigten bei einer mässigen physiologischen Excavation normale Färbung und Begrenzung.

Der Zustand blieb 6 Wochen lang unverändert bis zum 29. Juli. An diesem Tage wurde der Patient, der bis dahin gut lesen und schreiben konnte, plötzlich völlig blind. Eine Viertelstunde später bekam er Kopfschmerzen, musste sich erbrechen, und verlor auf kurze Zeit das Bewusstsein. Die Kopfschmerzen und das Erbrechen börten nach drei Tagen auf, die Blindbeit aber blieb bestehen. Noch längere Zeit fühlte er sicb elend und musste vier Wochen lang das Bett hüten.

Zwei Monate nach dem zweiten Anfall wurde er wieder untersucht. Er ist stark abgemagert und muss wie ein Blinder geführt werden; er kann nicht einen Schritt allein gehen. Zu seiner eigenen Ueberraschung stellt sich aber heraus, dass er beiderseits noch eine gute centrale Sebschärfe (r. von $\frac{2}{5}$, 1. von $\frac{5}{9}$ ) und normalen Farbensinn hat. Nur ist das Gesichtsfeld jedes Auges enorm verengt: es misst im horizontalen Durchmesser nur $1-1 \frac{1}{2}{ }^{0}$, im verticalen 2-2 $\frac{1}{2}{ }^{\circ}$. Der Patient kann aus diesem Grunde die vorgehaltenen Finger erst zählen, wenn sie $3-4 \mathrm{~m}$ von ihw entfernt sind, dagegen die feinste Druckschrift mit dem geeigneten Convexglase langsam, aber richtig lesen.

Auch jetzt bietet der objective Befund an den Augen absolut nichts Abnormes dar: keine Spur von Atrophie der Papillen, normale Reaction der Pupillen auf Licht, gleichviel ob man sie von der rechten oder linken Seite her beleuchtet, keinerlei Läbmung der Augenmuskeln und eben so wenig der Körper-Musculatur. Keine Störung der Sensibilität, kein Tremor der Zunge ołer der Hände; der Patient steht mit geschlossenen Augen sicher. In psychischer Beziehung macht er einen deprimirten Eindruck, der bei seiner Erblindung nir zu natürlich ist, aber seine Intelligenz ist erbalten; er gebietef über einen ziemlich reichen Wortschatz, und schreibt so gut, wie es ibn sein minimales Gesichtsfeld erlaubt. Auch das Gedächtniss für Thatsachen und Personen, sowie für die Einzelheiten seiner Krankbeit ist ihm trea geblieben. Dagegen ist seiner Frau aufgefallen, wie uberaus scblecht er sich im Raume orientirt. An sein Bett gefübrt, weiss er nicht, wo dessen Kopfende und dessen Fussende ist. In der Wohnung, die er seit vielen Jabren inne-hat, findet er nicht aus einem Zimmer in das andere. Er vermag nicht anzugeben, durch welche Strassen er aus seinem Hause nach der Klinik gegangen ist; alle topographischen Vorstellungen sind ihm abhanden gekommen, und er benimmt sich beim Geben viel ungeschickter, als ein total Blinder. Das geht so weit, dass er nicht im Stande ist, sich allein auf einen Stubl za setzen, den er mit den Händen betastet.

Die Diagnose wurde auf Grund ähnlicher Fälle, besonders wegen der Analogie, welche unser Fall mit dem von Foerster im Jahre $1890 \mathrm{im}$ Archiv für Ophthalmologie Bd. XXXVI, Abth. 1, S. 94-108 veröffentlichten 
darbot, auf eine ungefäbr symmetrische Erweichung der Rinde der medialen Flächen beider Occipital-Lappen in der Gegend der Fissura calcarina gestellt; als nächste Ursache der Erweichung wurden Embolien in die Arterien der Hinterbaupts-Lappen angenommen. Eine Läsion der Bulbi und der Sehnerpen, sowie der grossen HirnstammGanglien konnte wegen des normalen ophtbalmoskopischen Befundes und der Integrität der Pupillen-Bewegungen mit Sicherheit ausgeschlossen werden; demnach musste die Läsion weiter centralwärts in der Sebstrablung oder in der Rinde der Occipital-Lappen vermuthet werden. Die Abwesenbeit aller anderweitigen Cerebral-Erscheinungen nöthigte aber zu der Anuahme einer Läsion in der Rinde, und die successiven Anfälle von Hemianopsie zur Annahme einer beiderseitigen Läsion.

Alle angegebenen Störungen blieben unverändert, neue von Seiten des Gehirns kamen nicht hinzu. Im Mai 1898 litt der Patient viel unter schweren asthmatischen Anfällen; er bekam Ascites und Hydrops der unteren Extremitäten und starb in seiner Wohnung am 6. Juli plötzlich unter den Erscheinungen einer Herzlähmung.

Am 7. Juli hatte Herr Professor v. Recklinghausen die Güte, die Section der Schädelhöhle auszuführen, welche allein gestattet wurde. Das Gehirn wurde sofort in eine Formalinlösung und nach 3 Tagen in eine Mischung von Alkohol, Glycerin and Kali aceticum gebracht; nach 14 Tagen war es gut gehärtet und konnte makroskopisch untersucht werden. Herr Professor v. Recklinghausen constatirte Folgendes:

Die convexen Flächen beider Hemisphären zeigen zahlreiche Pacchioni'sche Granulationen, erschienen aber sonst normal. An der Basis zeigen sich die Gefässe theilweise atheromatös verändert. Das Chiasma und die Sehnerven bieten nichts Auffallendes dar. Bei der näheren Untersuchung der Arterien an der Basis findet sich links im zweiten Aste der Arteria cerebri posterior, der medianwärts verläuft, eine $4-5 \mathrm{~mm}$ lange Stelle, an der das Gefäss nicht znsammensinkt, und die kein Blut enthält. Die Stelle ist in der Figur 1 der Tafel XII durch einen Doppelpfeil bezeichnet. Durch Einführung einer Borste von der centralen Seite und einer zweiten von der peripherischen Seite her überzeugt man sich, dass biēr ein mehrere Millimeter langer, fester Verschluss besteht, der auch keine Luft durchtreten lässt ${ }^{2}$ ). Die Fortsetzungen des obliterirten Gefässes sind

3) Bei der genaneren Untersuchung findet sich an dieser Stelle ein $5 \mathrm{~mm}$ langer, rings mit der Wandung verwachsener, vollständig organisirter Thrombus. 
sämmtlich offen und grösstentheils bluthaltig. An der Carotis und der Arteria fossae Sylvii, welche relativ weit sind, finden sich sklerotische Stellen, ebenso an der Arteria basilaris, welche S-förmig gekrümmt ist.

An den Gefässen der Basis der rechten Hemisphäre wird ebenfalls nach einer Verschlussstelle gesucht, indem sie an einzelnen Stellen eröffnet und mit Luft gefüllt werden. Hier gelingt es jedoch nicht, an den Partien, die ohne Zerstörung des Präparates zugänglich sind, die obliterirte Stelle zu finden, die sehr peripherisch in der Tiefe gelegen sein muss. Ihre Constatirung bleibt einer weiteren Untersuchung vorbehalten, welche auch die Frage entscheiden wird, ob es sich um einen Thrombus oder um eine locale Sklerose handelt.

Die auffallendsten makroskopischen Veränderungen der Hirnsubstanz finden sich an der medianen, bezw. unteren Fläche beider Hemisphären.

In der linken Hemisphäre erscheint der ganze Cuneus eingesunken, stark abgeflacht, hellgelb und sehr weich, aber noch mit intacter Pia überzogen. Ferner ist der Gyrus lingualis und der Gyrus fusiformis ganz platt und erweicht und zwar beide in ganzer Ausdehnung (Tafel XII Figur 1). Nach vorn reicht die totale Erweichung bis zur Theilungsstelle der Art. profunda cerebri, da wo sie den zweiten und dritten Ast abgiebt. Auf diese Weise sind die beiden inneren Windungen der Unterfläche fast verstrichen und letztere so verdünnt, dass die untere Wandung des Hinterhorns des Seitenventrikels kaum noch 3-4 mm dick und wenn man von vorn in den Ventrikel hineinsieht, ganz durchscheinend ist.

Auch in der rechten Hemisphäre (Tafel XII Figur 2) ist der Cuneus erweicht und zerstört, aber nicht in seiner ganzen Ausdehnung; denn eine ansehnliche Portion des oberen Theils und der obere Rand nach der Convexität hin sind intact. Nach unten hin setzt sich die gelbe Erweichung fort in den hinteren Abschnitt des Gyrus lingualis bis $\frac{1}{2} \mathrm{~cm}$ von der Spitze des Occipital-Lappens entfernt, und nach vorn in einem langen Streifen längs des Randes, welchen der Gyrus lingualis der Fissura calcarina zukehrt, in einer Länge von $5 \mathrm{~cm}$ bis zur Stelle, wo 
die Art. prof. cerebri ihre Hauptäste abgiebt, d. h. sich in den dritten und vierten Ast theilt.

Alle übrigen Partien des Gehirns erscheinen nach gründlicher äusserer Besichtigung gesund.

Soweit der von Professor v. Recklinghausen erhobene Befund.

Zur weiteren Untersuchung wurden beide GrosshirnHemisphären von ihrem hinteren Ende an durch frontale, beiderseits jedesmal in gleichen Abständen geführte Schnitte in Scheiben zerlegt. Die braune Pigmentirung, sowie die Erweichung und Zerklüftung der erkrankten Partien ermöglichten im Allgemeinen eine genaue Abgrenzung derselben. Indessen wurde zur grösseren Sicherung die mikroskopische Untersuchung herangezogen, und zwar wurden die Scheiben, welche die für die Frage wichtigsten Regionen der Hirnoberfläche enthielten, oder einen Zweifel über die Ausdehnung der Erweichung aufkommen liessen, in toto in Celloidin eingebettet und in ganzer Fläche geschnitten, nehmlich links Scheibe 1, 2, 3, und der untere mediale Theil von 7, rechts Scheibe 1, 2 und 4 . Die Härtung des Gehirns nach Kaiserling's Methode schloss die Anwendung der Weigert'schen Markscheiden-Färbung aus. Indessen sind die Bilder, welche ungefärbte oder mit Hämatoxylin-Eosin behandelte Sohnitte darbieten, vollkommen ausreichend, um über den Zustand des Hirn-Parenchyms und die Grenzen der Zerstörung genauen Aufschluss zu geben.

Die Zeichnungen der Schnitte sind nach der makroskopischen Betrachtung der Scheiben und der Mikrotomschnitte gefertigt, und das Erweichungs-Gebiet durch dunkle Schraffirung hervorgehoben. Wo die mikroskopische Untersuchung weitere Aufschlüsse über die Ausdehnung des letzteren und das Vorhandensein kleinster Heerde ergab, wurden diese den Abbildungen hinzugefügt. Bei der Aufzeichnung and Beschreibung der Schnitte ist die Verbindungslinie zwischen dem oberen Hemisphärenrand und dem unteren lateralen Rand des Occipital-, bezw. Temporal-Lappens, welche thatsächlich schräg von oben nach unten und aussen zieht, als senkrecht von oben nach unten verlaufend angenommen worden, so dass die hauptsächlich zu besprechenden Theile, Cuneus, Fissura calcarina, Gyrus lingualis und Gyrus fusiformis, 
die mediale Hälfte des Schnittes einnehmen, während sie bei normaler Lagerung des Gehirns an der medialen-unteren Fläche des Hinterhaupts- and Schläfenlappens liegen. Es geschah eines Theils der Vereinfachung des Ausdruckes wegen, anderen Theils weil in den frontalen Schnitten der genannte Durchmesser in Folge der Abflachung der erweichten Gyri den der medialen unteren Fläche gegenüber dem senkrecht darauf stehenden, besonders linkerseits, bedeutend überwiegt und wie die natürliche Längsachse der Scheiben erscheint.

Die Theile der medialen und unteren Oberfläche des Occipital- und Temporallappens, welchen das Haupt-Interesse zufällt, sind der Cuneus, welcher durch die von hinten nach vorn laufende Cuneus-Furche in obere und untere Windung zerfällt, und dessen vordere untere Spitze sich zu dem Cuneus-Stiel auszieht; unter dem Cuneus, von hinten nach vorn ziehend, der Gyrus lingualis, und unter diesem der Gyrus fusiformis, beide von einander durch die Fiss. collateralis getrennt, mit ihren hinteren Enden aber zusammenfliessend. Ferner vor dem Cuneus, durch die Fiss. parieto-occipitalis von ihm getrennt, der Praecuneus; zwischen diesen und den Balken schiebt sich der Gyr. fornicatus ein, welcher den Balkenwulst umkreist, und unterhalb desselben sich mit dem Gyr. lingualis zum Gyr. hippocampi vereinigt. Die Verlängerung der Fiss. calloso-marginalis nach hinten trennt den Praecuneus vom horizontalen Theil des Gyr. fornicatus, der absteigende Theil des letzteren geht nach oben ohne Grenze in den Praecuneus über. Längs des oberen Randes des Caneus verläuft die Fiss. parieto-occip. zwischen ihm und dem Praecuneus, bezw. nach vorn zu dem absteigenden Theil des Gyr. fornic.; am Unterrand des Cuneus, zwischen ihm und Gyr. lingualis, die Fiss. calcarina; dieselbe schneidet tief von innen nach aussen ein, und besitzt einen flächenhaft ausgebreiteten Grund, an welchem die Rinde unmittelbar auf dem HemisphärenMark aufliegt; von der Stelle an, 'wo der Cuneus-Stiel unter die Oberfläche zurücktritt und vom Gyr. ling. und Praecuneus überwölbt wird, besitzen die Fiss. calcarina und Fiss. parieto-occip. einen gemeinsamen Eingang und nur in der Tiefe werden sie durch den Stiel getrennt; von dem Ende des letzteren an 
fliessen beide Furchen zu einem gemeinsamen Verlaufsstücke zusammen.

Beschreibung der Frontalschnitte.

A. Linke Hemisphäre.

Schnitt 1 (Taf. XIII L 1), $10 \mathrm{~mm}$ vor der Hinterhaupts-Spitze, $13 \mathrm{~mm}$ binter dem oberen Ende der Fiss. parieto-occip. Getroffen sind an der medialen Seite der Cuneus, durch die Cuneus-Furche (F. cun.) in 2 Windungen getheilt, ferner Gyr. lingualis (G. ling.) und Gyr. fusiformis (G. fus.), beide sehr platt und nicht durch eine Furche von einander geschieden (das untere Ende des G. fus. ist aus der Scheibe ausgebrochen). Zwischen G. ling. und unterer Cuneus-Windung schneidet die Fissura calcarina (F. calc.) ein, und breitet sich zu einem von beiden Windungen überwölbten, flächenbaften Grund aus. Die Erweichung der Hirnsubstanz beginnt am Grund der Cuneus-Furche, nimmt an der dieselbe begrenzenden oberen Cuneus-Windung nur einen subcorticalen Streifen ein, an der gegenüberliegenden unteren aber die Rinde selbst, und zieht, unter alleiniger Freilassung des oberen Randes der unteren Cuneus-Windung, an deren medialer Seite entlang bis zu ibrem unteren Rand, und zwar sind die genannten Theile stark braun gefärbt, weich und zerklüftet. An der dem Grund der F. calc. zugekehrten Seite der unteren Cuneus-Windung ist die Rinde etwas schwal, aber nicht erweicht; der Grund der F. calc. selbst besitzt eine vollkommen unveränderte, kräftige Rinde und rein weisse, in der Consistenz durchaus normale Marksubstanz. Die die. F. calc. begrenzende Fläche des Gyr. ling. ist, soweit sie dem Grund derselben gegenübersteht, in der Rinde und einer schmalen, angrenzenden Zone des Markes ebenfalls frei ron Veränderungen, dagegen in ihrem oberen, dem Cuneus gegenüber liegenden Abschnitt in der Rindenschicbt mit mebreren circumscripten Erweichungsheerdchen versehen bis zum oberen Rand hin, und an der medialen Seite des G. ling. und des G. fusif., also der abgeflachten Wölbung derselben entsprechend, ist die Substanz in $4 \mathrm{~mm}$ breiter, die ganze Rinde und den angrenzenden Theil des Marklagers in sich begreifender Schicht vollkommen erweicht, zerklüftet, braun gefärbt, und durch einen Spalt von dem normalen Gewebe lateralwärts abgegrenzt. Alle übrigen Theile des Schnittes sind unverändert. Die mikroskopische Untersuchung bestätigt diese Angaben über die Ausdehnung der Erweichung, namentlich das Intactsein des Grundes der Fiss. calcarina, zeigt aber ferner in der der letzteren zugekebrten Rinde der unteren Cuneus-Windung einige kleinste Heerde, innerhalb deren die Glia durchbrochen erscheint durch runde Lücken, zwischen denen ibre Fäden ausserordentlich deutlich, weit von einander isolirt, hervortreten. An-den Stellen vollkommener Erweichung lässt sich mikroskopisch die Grenze von Rinde und Mark in der Regel noch nachweisen: Die Rinde neholich ist umgewandelt in eine sehr körnige, obwohl ganz dichte und durch Hämatoxylin stark blau gefärbte Substanz, welche durch zahlreiche Spalten zu wolkigen Ballen verschiedener Grösse zerklüftet ist und, selbst kernlos, in diesen 
Spalten zahlreiche runde Zellen einschliesst, welche zum Theil kleine, oft mebrkernige Leukocyten, meist aber grosse Elemente darstellen, die alle die bekannten Eigenschaften der Körnchenkugeln im gehärteten Zustand aufweisen; in einzelnen der Spalten laufen capilläre Blutgefässe, theilweise mit rothen Blutkörperchen gefïllt. Ganglien-Zellen fehlen fast vollständig, ganz selten kommt inmitten eines Ballens eine solche in hyalin verändertem $\mathrm{Zu}$ stand vor als sehr grosses, ovales Gebilde mit excentrisch gestelltem Kern und homogenem Protoplasma. Dagegen besteht das erweichte Mark lediglich aus Körnchenkugeln, welche, lose liegend, die weiten Maschen eines aus dünnwandigen Blutgefässen gebildeten Netzes unvollkommen füllen. So erscheint die Marbsubstanz in gefärbten Schnitten viel heller, als die einen dunkeln Saum bildende Rinde. Auf letzterer liegt oft noch eine Schicht von Körnchenkugeln dadurch, dass dieselben die Maschen der weichen Häute füllen, sicherlich durch Resorption aus den Erweichungs-Heerden dahin gelangt, und am dicbtesten um die grösseren Gefãsse liegend. Der Rindensaum ist an den so veränderten Partien regelmässig bedeutend verschmälert, selten fehlen an einzelnen Punkten die die Stelle der Rinde bezeichnenden. dunkeln Ballen ganz, und die Körnchenkugeln der weichen Häute gehen unmittelbar in die der Marksubstanz über. Die Möglichkeit, auf die angegebene Weise noch die Grenze von Mark und Rinde in den ErweichungsHeerden festzustellen, war bei allen folgenden Schnitten, namentlich denen der rechten Hemisphäre, von grossem Vortheile.

Scbnitt 2 (Abbild. L 2), $18 \mathrm{~mm}$ vor der Hinterbaupts-Spitze, $5 \mathrm{~mm}$ hinter dem oberen Ende der Fiss. parieto-occipitalis. An der medialen Seite fallen in den Schnitt der Cuneus mit seinen 2 Windungen und der Cuneus-Furche, Fiss. calcarina, Gyrus lingualis und Gyrus fusiformis, die beiden letzteren durch die Collateral-Furche (F. coll.) von einander getrennt. Die Fiss. calcarina schneidet tief von oben nacb unten ein, besitzt nur eine mediane und eine laterale Wand, aber scheinbar keinen flächenhaft ausgebreiteten Grund. Indessen lässt die continuirliche Verfolgung der Furcho nach hinten und nach vorn erkennen, dass das, was auf Scbnitt 2 und auch auf den nach vorn zu folgenden sich als laterale Wand darstellt, in ihren unteren zwei Dritteln dem Grund der Fissur auf Schnitt 1 entspricht, welcher in Folge des Zusammensinkens der sonst überhängenden Cuneus-Windung jetzt in seiner ganzen Ausdehnung der vom Gyrus lingualis gebildeten Wand gegenüberstebt. G. lingualis und G. fusiformis sind ganz abgeplattet und dadurch bedeutend verbreitert, die mediale Fläche, also der Kamm der unteren Cuneus-Windung, stark eingesunken; indessen überlagert der untere Rand der letzteren den oberen Rand des G. ling. noch etwas, so dass der Eingang zur Fiss. calc. nicht klafft. $1-2 \mathrm{~mm}$ unter der Oberfläche der unteren Cuneus-Windung im Bereich der Marksubstanz zieht ein Spalt als laterale Grenze der Erweichung; derselbe läuft oben in die Marksubstanz der oberen Cuneus-Windung aus, und endet unten $3 \mathrm{~mm}$ über der F. calc. Alles, was median von demselben liegt, ist stark braun pigmentirt und voll- 
kommen erweicbt, nebmlich der innere Theil der Basis der oberen CuneusWindung und die 2. Cuneus-Windung in ihrer ganzen Breite. Weiterbin sind erweicht und durch zablreiche Spaiten zerklüftet die Rinde und die angrenzende Marksubstanz an der medialen Fläche des G. ling., mit Ausnahme des dem Cuneus gegenüberstehenden Oberrandes (in der Zeichnung ist irrthümlich dieser letztere mit als erkrankt schraffirt), und die Erweichung setzt sich nach unten continuirlich auf den $G$. fusiforinis fort, an diesem tiefer in die Marksubstanz hineingreifend, als am G. ling., und ihn in seiner ganzen Höben-Ausdehnung zerstörend. Verschont von der Erweichung sind aber die mediale, vom Gyr. ling. gebildete Wand der Fiss. calcarina, die Unbiegungsstelle derselben in die laterale $W$ and, der eigentliche Grund der Fissur und das untere Drittel der lateralen Wand. Und zwar ist in der ganzen genannten Ausdehnung die Rinde durchaus fest, in Farbe und Breite nicht unterschieden von der Rinde an der convexen Oberfläche des Gehirns, ferner an der medialen Wand der Fissur die Marksubstanz des G. ling. in einer Breite von $1 \mathrm{~mm}$ erbalten, an der lateralen Wand, dem Grund der Windung aber, das Mark durch die ganze Breite der Hemisphäre bis zur Convexität vollkommen normal. Die mikroskopische Untersuchung ergiebt, dass in der die Fiss. cale. begrenzenden Rinde des G. ling. der obere Abschnitt dicht ober- und dicht unterbalb der auch in der Figur angegebenen kleinen Furche je einen kleinsten, punktförmigen Erweichungs-Heerd enthält, in der ganzen übrigen Ausdebnung aber, und an der Umbiegungs-Stelle zu dem Grund der Fissur, sowie dem unteren Drittel des letzteren durchaus unveränderte Structur besitzt; ferner erweist sich die dazu gehörige Marksubstanz in der früher bezeichneten Ausdehnung auch bei mikroskopischer Betrachtung normal; nur sebliessen sich an das untere Ende des ErweichungsSpaltes im Cuneus bis zu der Rinde am Grunde der F. calc. mebrere kleinste Erweichungs-Heerdchen an, ohne indessen in das zu den normalen Rindentheilen des letzteren gebörige Markgebiet überzugreifen.

Schnitt 3 (Abbild. L 3), $24 \mathrm{~mm}$ vor der Hinterhaupts-Spitze, dureh den oberen Endpunkt der Fiss. parieto-accipitalis gelegt. Er trifft den vorderen Theil des Cuneus, an welchem die obere Windung sebr schmal ist, die Fiss. calcarina, Gyr. ling. und Gyr. fusif. mit der Collateral-Furehe dazwischen, aus deren klaffendem Eingang eine kleine Erhebung ihres Bodens an die Oberfläche ansteht. Auf dem lateralen, grösseren Theil des Schnittes ist wiederum Alles intaet. Die Erweichung besehränkt sich auf die medialen Partien, reicht hier aber von der unteren Cuneus-Windung continuirlich nach abwärts bis zum tiefsten Punkt des Schnittes, dem unteren Rand des G. fusiformis. Auch hier bildet ein fast unanterbrochener Spalt die Grenze z.wischen gesundem und krankem Gewebe: Derselbe beginnt am Grund per Cunens-Furche, und steigt nach unten, immer in der Marksubstanz, nach aussen vom G. ling. und G. fusif, um auf die tiefste Einsentung der den letzteren lateral begrenzenden Furche zu stossen. An der unteren CuneusWindung gemessen liegt er $2 \mathrm{~mm}$, in der Mitte des G. ling., $3 \mathrm{~mm}$ von der 
medialen Oberfläche entfernt. Er scheidet also von dem vollkommen normalen äusseren Abschnitt des Occipital-Lappens die untere Cuneus-Windung, den G. ling. und G. fusif. in ibrer ganzen Höhen-Ausdehnung, und nach einen Streifen der darunter liegenden Marksubstanz des Centrum semiovale ab, und diese genannten Theile sind bis auf 3 kleine Bezirke vollkommen erweicht und zerklüftet, intensiv braun gefärbt, und die Gyri, wie die angegebenen Maasse schon aussagen, ganz beträchtlich abgeplattet. Die Grenze ron Rinde und Mark ist in den erweichten Partien vollkommen unkenntlich. Aus diesem veränderten Gebiet hebt sich der obere Rand und die der Fiss. calcarina angebörige Wand des G. ling. dadurch bervor, dass sie nicht zerklüftet, nicht braun gefärbt sind und Rinde und Mark unterscheiden lassen; indessen nur makroskopisch; mikroskopisch finden sich sowohl in der Wand der Fiss. calcar., Rinde und Mark, als auch in der Umbiegung derselben zur medialen Oberfläche des Gyrus die Rinde von einer ganzen Zahl kleinster Erweichnngs-Heerdchen durchsetzt, und nur eine $7 \mathrm{~mm}$ lange Strecke der Rinde an der medialen Oberfläche, also auf dem Kamm der Windung, ist auch mikroskopisch unverändert. Zweitens ist in der Structur erhalten die oberflächliche Rindenpartie des Bodens der Fiss. collateralis, und drittens die Rinde am tiefsten Punkt, d. b. dem unteren lateralen Rand des G. fusiformis. In das Bereich der Erweichung fällt also auch die Rinde und Marksubstanz an der lateralen Wand, dem eigentlichen Grund der Fiss. calcarina.

Schnitt 4 (Abbild. L 4), $31 \mathrm{~mm}$ vor der Hinterbaupts-Spitze. Trifft an der medianen Seite den Praecuneus ( $\mathrm{Pe}$ ), den als flacher Höcker hervortretenden Cuneus-Stiel (Cs), zwischen beiden die flache Fiss. parieto-occipitalis, ferner die von oben nach unten einschneidende Fiss. calcarina und den Gyr. lingualis und Gyr. fusiformis. Die Fiss. collateralis schneidet nicht wie an den früheren Schnitten ein, sondern die Grenze zwischen den beiden vollikommen abgeplatteten Windungen wird nur durch eine ganz leichte Einsenkung der Oberfläche bezeichnet, in welcher die in Schnitt 2 und 3 in der Fissur verlaufende Arterie (Art. c) liegt. Der oberste Abschnitt der medialen Praecuneus-Fläche ist unverändert; die Erweichung beginnt in der Mitte der letzteren, zieht, an der medialen Seite immer bis zur Oberfäche reichend, nach abwärts und wird, wie früher, durch einen fast continuirlichen Spalt gegen den normalen äusseren Theil des Schnittes begrenzt. Dieser Spalt verläuft durebweg in der weissen Substanz der Hemispbäre, ist von der medialen Oberfäche, sowobl entsprechend dem oberen Absehnitt des G. ling. als dem unteren des G. fusif., $7 \mathrm{~mm}$ entfernt. Nur entsprechend der Grenze dieser beiden Windungen ist der Spalt unterbrochen, aber ersetzt durch eine scharfe Grenzlinie 2 wischen gesunder und erkrankter Substanz; von der Fiss. calcarina ist der Spalt $3 \mathrm{~mm}$ entfernt. Alles, was median von ibm, bezw. jener ihn unterbrechenden Grenzlinie liegt, ist total zerstört: Wie die angeführten Maasse aussagen, ist die gesammte betreffende Partie stark zusammengesunken, die Höhe der Windungen be- 
deutend reducirt, ibre Oberfläche abgeplattet, die Substanz braun gefärbt; ganz scblaff, stark zerklüftet, und die Grenze der Rinden- und Marksubstanz vollständig geschwunden. Betroffen sind von dieser Veränderung also die untere Hälfte des Praecuneus, der Cuneus-Stiel, der G. ling. und G. fusif. in ganzer Ausdehnung, and der unter allen diesen Windungen liegende Abschnitt des Marklagers der Hemisphäre. Die Fiss. calcarina fällt demnach in's Bereich der Erweichung, ihre gesammte Rinden-Auskleidung ist betheiligt und die unterliegende Marksubstanz auf der lateralen Seite, dem eigentlichen Grund der Fissur, bis zu dem erwähnten Erweichungs-Spalt, auf der medialen durch die ganze Dicke des G. ling. Von der Grenze zwischen Rinde und Mark in der Wand der Furche ist noch eine Andeutung $z \mathfrak{u}$ erkennen, und $z w a r$ ist die Rinde noch ziemlich steif, indessen bis auf $\frac{1}{2} \mathrm{~mm}$ verschmälert und gelb gefärbt, die angrenzende Marksubstanz aber vollkommen erweicht.

Schnitt 5, $38 \mathrm{~mm}$ vor der Hinterhaupts-Spitze. Er trifft das gemeinsame Verlaufsstück der Fiss. calcarina und Fiss. parieto-occipitalis, der Cuneus-Stiel endet $4 \mathrm{~mm}$ hinter der Schnittfläche. So stellt die Fissur einen von oben nach unten verlaufenden Einschnitt dar, dessen wediale Wand vom Gyrus lingualis gebildet wird, während die laterale, eigentlich der Grund der Furche, nach oben unmittelbar sich in die mediale Fläche des Praecuneus fortsetzt; letzterer wölbt sich nicht über den Eingang zur Fissur über, erhebt sich überbaupt nicht an der medialen Oberfläche, sondern ist stark abgeflacht. Ebenso sind der G. ling. und der wiederum nur durch die Art. collateralis gegen ihn abzugrenzende G. fusiformis ganz platt. Der G. ling. bildet ein aufsteigendes Läppehen von $2,5 \mathrm{~mm}$ Dicke, und in seiner Axe, also an Stelle der Marksubstanz, liegt ein Spalt, während die begrenzende, jederseits $1 \mathrm{~mm}$ breite Rindenschicht ganz schlaff, weich und braun ist. Am G. fusiformis lässt sich keine Rindensubstanz unterscheiden; die Pia liegt unmittelbar auf der Marksubstanz, die nur an der Oberfläche gelb gefärbt, im Uebrigen unverändert ist. An der lateralen Wand, dem Grund der Fiss. calcarina, fehlt die Rinde vollkommen, auch bier folgt unter der Pia mater sofort die Marksubstanz, ebenfalls nur an ibrer Oberfläche braun pigmentirt. Nach oben zu, am Uebergang zum Praecnnens, kommt unter der Pia eine etwa $\frac{1}{2} \mathrm{~mm}$ dicke, durch einen Spalt von der angrenzenden Hirnsubstanz abgehobene, schlaffe, braune Lamelle, jenseits des Spaltes liegt ebenfalls braun gefärbte Marksubstanz. Dieser Spalt gehört der unteren Hälfte des Praecuneus an, in der oberen Hälfte sind Mark und Rinde durchaus unverändert, ebenso in allen äbrigen Theilen des Schnittes.

Schnitt 6 (Abbild. L 6), $45 \mathrm{~mm}$ vor der Spitze des Occipital-Lappens durch das hintere Ende des Binterhorns gehend. An der medialen Seite der Praecuneus und unter ibm, durch die Fiss. calloso-marginalis getrennt, die flach getroffene Umbiegungsstelle des Gyr. fornicatus (G. forn.), ferner G. ling., G. fusif. mit der als kleiner Einscbnitt kenntlichen Fiss. collateralis. An der Fiss. calcarina, bezw. dem gemeinsamen Verlaufsstück derselben mit 
der Fiss. parieto-occip. ist der flächenhafte Grund wieder deutlicher ausgesprochen dadurch, dass er gegen den $G$. fornic. durch eine kleine Fissur geschieden wird; jedoch liegt die von letzterem gebildete Wand der Fissur fast in einer schräg aufsteigenden Linie mit dem Boden, und die vom G. ling. gebildete mediale Wand steht beiden gegenüber. Der G. fornic. ist völlig unverändert, aber unmittelbar unter seiner Basis liegt im Hemisphären-Mark ein 2,5 mm langer und $1 \mathrm{~mm}$ breiter Erweichungs-Heerd. Am ganzen Grund der Fiss. calcar. ist die Rinde stark verschmälert, gelb und erweicht, das Mark darunter aber intact, der vom Gyr. ling. gelieferte Theil der Wand aber ist total erweicht, und zwar reicht die Zerstörung durch die ganze, kaum $2 \mathrm{~mm}$ betragende Dicke des Läppchens, als welches sich, wie im vorigen Schnitt, der Gyrus darstellt. Axial ist dasselbe wiederum hohl. Die veränderte Substanz des G. ling. gebt nach unten über in eine äusserst schmale, stark braune Gewebsschicht, welche an Stelle des Gyr. fusit. zwischen Pia mater und Ependym liegt; die Region dieses Gyrus setzt sich noch unterhalb des Hinterhorns auf der medialen Seite des Schnittes fort, auch hier vertreten durch einen ganz scbmalen, total zerstörten Gewebsstreif. Sowobl der Praecuntus, als alle Theile lateral vom Hinterhorn sind durchaus unverändert.

Schnitt 7, $52 \mathrm{~mm}$ vor der Hinterhaupts-Spitze. An der medialen Seite erscheinen untereinander Lobus paracentralis, Praecuneus, Gyr. fornicatus und, von diesem durch die Fiss. calcarina in ihrem gemeinsamen Verlaufsstück mit der Fiss. par.-occip. getrennt, der Gyr. lingualis, darunter der Gyr. fusiformis - die Collateralfurche seicht - und die 3. Schläfenwindung. Das Erweichungs-Gebiet betrifft wiederum G. ling., G. fusif, und den Grund der Fiss. calcarina. Die beiden genannten Windungen sind total zerstört und besitzen dieselbe Gestalt, wie in Schnitt 6, d. b. der G. ling. bildet ein $1,5 \mathrm{~mm}$ breites, schlaffes, braunes Läppchen mit einem axialen Spalt; letzterer setzt sich nach unten in die $1,5 \mathrm{~mm}$ breite braune Gewebsschicht fort, welche an Stelle des G. fusif. zwischen Pia und Ependym liegt; an dieser Stelle buchtet sich das Hinterhorn ziemlich stark über die mediale Fläche vor; unterhalb desselben bildet der G. fusif. weiterhin eine dünne, schlaffe, braune Gewebssebicht. Die angrenzende 3. Temporalwindung dagegen ist unverändert. An der oberen äusseren, dem G. fornic. zugehörigen Wand der Fiss. calcarina ist die Rinde durebaus normal, von der darunter liegenden Marksubstanz aber nur ein schmaler, durehschnittlich $1 \mathrm{~mm}$ breiter Streifen; nach aussen und oben von diesem folgt im Mark der Windung eine ganze Zabl von nur im mikroskopischen Präparat sichtbaren, kleinsten Erweichungs-Heerden, weiter nach oben aber ein grösserer, welcher sich unter der Basis des Praecuneus nach aussen in's Marklager der Bemisphäre $6 \mathrm{~mm}$ weit erstreckt. Der Grund der Fiss. calcar., welcher hier wieder deutlich ausgesprochen ist, scheint makroskopisch ganz zerstört, während sich mikroskopisch eine ganz schmale, subpiale Zone der Rinde erhalten zeigt; von dieser an reicht die Erweichung bis an's Ependym heran. An 
der Umbiegung des Grundes der Furcbe in die obere äussere Wand liegt in der mittleren Schicht der Rinde eine Gruppe pyramidaler Ganglienzellen, welche bis in ibre Fortsätze hinein verkalkt sind. Einzelne mikroskopisch kleine Erweichungs-Heerde finden sich lateral vom Hinterborn in der unmittelbar angrenzenden weissen Substanz, die übrigen Regionen des Scbnittes sind normal.

Schnitt 8, $59 \mathrm{~mm}$ vor der Spitze des Occipital-Lappens, führt durch das Splenium corporis callosi. Die äussere Wand des Hinterhorns ist gleichmässig getrümmt, die innere bildet ein Knie; unterbalb desselben ist die Wand ganz dünn und durchscheinend, weil das Ependym und die Pia mater, beide stark braun gefärbt, ohne zwischengelagerte Hirnsubstanz unmittelbar einander berühren, während oberhalb des Knies medianwärts vom Ventrikel, dicht unterhalb des Balkens, der Gyr. hippocampi als $5 \mathrm{~mm}$ boher Lappen emporsteigt. Seine mediale Fläche besitzt eine schmale gelbe Rinde, und fällt nach unten $a b$ in den durchscheinenden Theil der Ventrikelwand unterhalb des Knies. Unterbalb des Ventrikels besitzt die mediale untere Oberfläche bis zur Basis der 3. Schläfenwindung keine deutliche Rinde, sondern unter der Pia mater einen lineären braunen Saum, an den sich unmittelbar Marksubstanz schliesst. Dieser Theil und die durchscheinende Partie der Ventrikelwand entsprechen dem G. fusiformis; das vordere Ende des G. lingualis liegt in der Scheibe zwischen Schnitt 7 und 8 . Alles über dem Balken und nach aussen vom Hinterborn Gelegene ist vollkommen nuverïndert.

Schnitt 9, $64 \mathrm{~mm}$ vor der Spitze des Occipital-Lappens, durch den hinteren Theil des Balkens und den hinteren Fornix-Schenkel fübrend. Die Erweichung hat in der Höhen-Ausdehnung abgenommen, betrifft nur den in seinem vordersten, verjüngten Ende getroffenen G. fusif. und den demselben zugekehrten Theil des G. hippocampi; eine Grenze zwischen beiden Windungen ist indessen nur durch die Arterie markirt, die Furche ist vollkommen verstrichen. Der G. fusif. ist total zerstört, an seiner Stelle nur eine dünne Schicht zerklüfteter, brauner Substanz zwischen Pia und Ependym vorhanden. An der medialen unteren Fläche des G. hippocampi fehlt die Rinde, die Pia liegt anf der in der oberfächlichsten Schicht braun gefärbten Marksubstanz.

Scbnitt 10,70 $\mathrm{mm}$ vor der Hinterhaupts-Spitze. Der Gyr. fusif. ist nicht mehr vorhanden, sein Ende liegt zwischen Sehnitt 9 und 10. Die 3. Schläfenwindung ist unverändert, dagegen der Pia-Fortsatz, welcher in die Furche zwischen ihm und dem Gyr. hippocampi herabsteigt, ganz gelbbraun pigmentirt, and ebenso die Oberfläche des letzteren; von seinem Wurchschnitt ist nur die die genannte Furche begrenzende Rinde and ein Theil der darunter liegenden Marksubstanz intact, der übrige Theil aber gelb and erweicht.

Schnitt 11, $76 \mathrm{~mm}$ vor der Hinterbaupts-Spitze, hart hinter dem Grosshirnschenkel gefübrt. Er zeigt nur einen ganz circumscripten, gelben, stark erweichten Heerd am medialen Rand des Gyr. hippocampi, welcher von der 
Rinde an durcb die Marksubstanz nach der medialen Kante des Hinterhorns bis hart ans Ependym heran als ein $3 \mathrm{~mm}$ breiter Streifen reicht; alle übrigen Theile zeigen normales Verbalten.

Scbnitt 12, $82 \mathrm{~mm}$ vor der Hinterhaupts-Spitze durch den Grosshirnschenkel gehend, lässt an dem dem letzteren zugewandten breiten Rand des Gyr. hippocampi einen kleinen Sulcus erkennen, in dessen Tiefe die Rinde in der Ausdehnung von ca. $3 \mathrm{~mm}$ stark gelb und weich ist. Es ist dies der einzige Erkrankungs-Heerd auf dem Schnitt.

Schnitt 13, $87 \mathrm{~mm}$ vor der Spitze'des Occipital-Lappens, $18 \mathrm{~mm}$ hinter dem vordersten Punkt des Uncus, zeigt durchaus normale Verbältnisse, auch an der noch vorhandenen Furche des Gyr. hippocampi keine besondere Färbung und keine Erweichung.

In der ganzen übrigen Hemisphäre, speciell in den Stammganglien findet sich Nichts von anderen Erkrankungs-Heerden.

\section{B. Recbte Hemisphäre.}

Schnitt 1, $10 \mathrm{~mm}$ vor der Spitze des Oceipital-Lappens. Der Cuneus zerfällt durch die Cuneus-Furche in obere und untere Windung; Gyr. lingualis und Gyr. fusiformis geben obne äussere Grenze in einander über. Alle diese Theile sind wesentlich stärker gewölbt, weniger abgeflacht, als an der linken Hemisphäre, sodass der breite Durchmesser des Scbnittes $18 \mathrm{~mm}$ gegen $13 \mathrm{~mm}$ links beträgt. Die Fissura calcarina entfernt sich dementsprechend auch weiter von der medialen Oberfläche des Gyr. lingualis, als links. Sie besitzt auf dem Schnitt die Gestalt eines S, dessen Axe von innen nach aussen und etwas nach Unten läuft, und dessen innere Krümmung die Concavităt nach oben kehrt; das Schlussstück der Furche, der Grund, läuft ziemlich gerade nach abwärts. Die Fissur liegt mit ibrer ganzen Ausdednung inmitten eines grossen Erweichungs-Bezirkes: Lateralwärts wird derselbe gegen das gesunde Gewebe begrenzt durch einen Erweichungsspalt, welcher aus der weissen Substanz der oberen Cuneus-Windung herabkommt, $2 \mathrm{~mm}$ nach aussen vom Grund der Fiss. calcar. vorüberläuft, und schliesslich in die Tiefe der Furche zwischen Gyr. fusif. und 3. Occipital-Windung einmündet, sodass also der mediale und laterale Theil des Schnittes nur noch durch die Rinde der oberen Cuneus-Windung zusammengehalten werden. Der mediale Theil ist fast vollkommen zerstört: Im Nivean des Schnittes stehen nur die Rinde des G. fusif., die Wand der Fiss. calcarina als ein durchschnittlich $1 \mathrm{~mm}$ breiter Saum gelbbraun pigmentirter Substanz, ferner die Rindentheile an der medialen Seite des Gyr. ling., und die des ganzen G. fusif., an letzterem bestebend aus unveränderter grauer Substanz, am G. ling. dagegen etwas schmal und von einzelnen Einsenkungen unterbrochen, und endlich steht in der Auskleidung der Cuneus-Furche die gelb gefärbte und stark verschmälerte Rinde. Alle übrigen Theile sinken unter die Schnittfläche zurück, bestehen aus ganz schlaffem, zerklüftetem Gewebe von brauner Färbung; im G. ling. und dem angrenzenden Theil des G. fusif. liegt sogar an Stelle der Marksubstanz eine Cyste, welche bis an die stehen- 
gebliebene mediale Rinde heranreicht, von der Rinde der Fiss. calcar. noch durch eine $1 \mathrm{~mm}$ breite Schicht schlaffen Gewebes getrennt bleibt, und mit dem aus dem Cuneus herabsteigenden Erweichungs-Spalt in Verbindung steht; ferner ist im unteren Abschnitt der 2. Cuneus-Windung neben jenem Spalt eine Höble yorhanden, welche fast unmittelbar bis zur Rinde der Fiss. calcarina reicht, so dass also die Wandung der letzteren fast völlig aus der umgebenden Marksubstanz herausgelöst ist. Im oberen Abschnitt der 2. Cuneus-Windung sind das Mark und die mediale Rinde ganz schlaff, weich und braun. Die mikroskopische Untersuchung zeigt, dass im Cuneus der Spalt $2 \mathrm{~mm}$ unter der Rinde verläuft, dass ferner die Rinden-Auskleidung der ganzen Fiss. calcarina nur $\frac{1}{2}-\frac{3}{4} \mathrm{~mm}$ breit und in ihrer Structur völlig zerstört ist, und dass die schmale Schicht von Marksubstanz, welche daran hängt, nur aus einem lockeren Gefässgerüst mit eingelagerten Körnchenkugeln bestebt, dass endlich an der medialen Seite des Gyr. ling. die stehengebliebene Rinde an mehreren Punkten von kleinen, aber die ganze Dicke der Rinde einnehmenden ErweichungsHeerden durchsetzt ist.

Schnitt 2 (Abbild. R 2), $18 \mathrm{~mm}$ vor der Hinterhaupts-Spitze geführt. Er trifft an der medialen Seite die 2 Cuneus-Windungen, den Gyr. ling., und den von diesem durch eine tief einschneidende Collateral-Furche getrennten Gyr. fusif.; der G. ling. besitzt eine kleine Furche. Der Eingang zur Fiss. calcar. klafft weit, auf $5 \mathrm{~mm}$, da sowohl die vom G. ling., als die rom Cuneus gebildete Wand stark eingesunken ist. Die Fortsetzung der Furehe ist mit blossem Auge gar nicht zu unterscheiden, denn sie fällt in das Bereich eines Erweichungs-Heerdes, welcher in seiner ganzen Ausdehnung gleichmässig aus intensiv braun gefärbter, sehr schlaffer und zerklüfteter Substanz besteht. Der Heerd reicht von dem oberen Abschnitt der medialen Oberfläche aus wie ein Keil nach unten in die Marksubstanz der Hemisphäre hinein, und ist sehr scharf gegen die gesunde Substanz abgegrenzt; lateralwärts sogar durch einen Spalt; der letztere beginnt auf dem höchsten Punkt der oberen Cuneus-Windung, läuft nach unten und etwas nach innen durch diese Windung und das Hemispbärenmark, und trifft inmitten des letzteren mit der medialen Grenzlinie des Heerdes zusammen, an welcher gesundes und krankes Gewebe noch zusammenhängt. Diese Grenzlinie beginnt an der medialen Oberfläche, also auf dem Kamm des Gyr. lingualis, und läuft schräg nach unten und aussen; die Spitze des Erweichungs-Heerdes liegt $8 \mathrm{~mm}$ unter dem klaffenden Theil der Fiss. calcarina, und somit fällt in das Erweichungs-Gebiet der mediale Abschnitt der oberen Cuneus-Windung, die ganze antere Cuneus-Windung, der obere Theil des G. ling., die ganze. Wand der Fiss. calcarina. Ueber die Tiefenausdehnung des Heerdes, besonders in der Umgebung der letzteren, geben die mikroskopischen Präparate schon bei Betrachtung mit blossem Auge Aufschluss insofern, als sie die Rinde der gesanmaten Fissur als einen sebr schmalen blau gefärbten Streifen erkennen lassen: Der Verlauf der 
Furche von dem klaffenden Eingang an gestaltet sich so, dass sich ein nicht erweitertes, $2 \mathrm{~mm}$ langes Stück anschliesst, und zu dem flächenhaft ausgebreiteten Grund der Fissur führt, welcher, von oben nach unten und etwas nach innen verlaufend, eine Ausdehnung von $7 \mathrm{~mm}$ bat. Die ganze Rinden-Auskleidung der Furche ist in der früher beschriebenen Weise zerstört; medianwärts beträgt die Breite der anschliessenden, erweichten Markzone $2 \mathrm{~mm}$, lateral $1,5 \mathrm{~mm}$, und somit ist die gesammte graue und weisse Substanz der Wand der Furche zerstört. Alle übrigen Theile des Schnittes ausserhalb des beschriebenen Heerdes sind unverändert.

Schnitt 3 (Abbild. R 3), $24 \mathrm{~mm}$ vor der Hinterhaupts-Spitze; getrofen sind clieselben Theile, wie auf Schnitt 2. Die Berührungsflächen der unteren Cuneus-Windung und des Gyr. ling. sind so stark eingesunken, dass der Eingang zur Fiss. calcarina wiederum klafft. Der Erweichungs-Heerd besitzt dieselbe Localisation, Form und Ausdehnung, wie auf Schnitt 2: Er liegt wieder in der Umgebung der Fiss. calcarina, begreift die Wandungen derselben vollkommen in sieh, reicht als Keil in die weisse Substanz hinein, und wird lateral durch einen Spalt begrenzt, der in das Mark der oberen Cuneus-Windung eintritt, und dicht unter der Rinde derselben endet. Auch die mediale Grenze ist durch einen Spalt bezeichnet, der aus dem Hemisphärenmark durch die weisse Substanz des G. lingualis bis zu dessen eingesunkener Oberfiäche zieht. So ist also hier der Erweichungs-Heerd, wie ein Sequester, fast vollkommen aus der Umgebung ausgelöst, und wird in seiner Lage nur durch die Pia mater und die Rinde auf dem Kamm des Cuneus erhalten. Die Fiss. calcarina selbst ist als feiner Spalt zu verfolgen und bildet die Axe des Erweichungs-Kegels, ihre Rinden-Bekleidung und zugehörige Marksubstanz ist aber allseitig und in derselben Ausdehnung, wie auf Schnitt 2, total zerstört. Wo der Grenzspalt der Erweichung die Oberfläche des G. ling. erreicht, lässt die letztere keine Rinde erkennen, vielmehr liegt die braun pigmentirte Pia unmittelbar auf der an der Oberfläche gelb gefärbten Marksubstanz. Dagegen sind an der die Fiss. collateralis begrenzenden Fläche der Windung Rinde und Mark unverändert, ebenso der ganze G. fusif. und alle Theile an der Convexität.

Schnitt 4, $31 \mathrm{~mm}$ vor der Spitze des Occipital-Lappens. $\mathrm{Zu}$ den im vorigen Schnitt getroffenen Theilen kommt die hinterste Spitze des Hinterborns, welche als ein $1,5 \mathrm{~mm}$ breiter Spalt sich darstellt. Der braune, keilförmige Erweichungs-Heerd liegt genau an derselben Stelle, wie in Schnitt 3; der denselben lateral begrenzende Spalt läuft im Marke der ersten Cuneus-Windung nach abwärts, dann durch das Hemisphären-Mark auf die Hinterhorn-Spitze zu, und endet unmittelbar über der letzteren; der mediale Grenzspalt zieht von hier nach innen durch den G. ling. hindurch bis zur Oberfläche, so dass wiederum eine untere, der Fiss. collateralis zugewandte Hälfte des Gyrus unverändert ist, während die obere in den Erweichungs-Heerd fällt. Der letztere umfasst also dasselbe Gebiet, wie in den früheren Schnitten, d. h. ausser dem genannten Abschnitt des G. ling., 
den ganzen Cuneus, an welchem lediglich einige Rindenbezirke erbalten sind, nehmlich an der medialen Seite der ersten Windung und an dem Oberrand der zweiten Windung, während das darunter liegende Mark zerstört ist, und ferner Rinde und Mark am Grund der Fiss. calcarina. Also ist wieder die gesammte Wand der letzteren zerstört und durch die zwei Spalten aus der Umgebung ausgelöst. Der Verlauf der Fissur selbst ist in den mikroskopischen Schnitten leicht festzustellen: Sie zieht, an der Oberfäche weit klaffend, dann einen engen Spalt bildend, nach innen, und biegt dann unter spitzem Winkel nach oben um. Dieser aufsteigende Schenkel entspricht dem Grund der Furche. Mikroskopisch findet sich auch ausserbalb des lateralen Spaltes noch eine 1-2 $\mathrm{mm}$ breite Zone des Hemispären-Marks zerstört, obschon nicht total verflüssigt, und auch die Hinterhorn-Spitze ist ringsherum von erweichter Substanz umgeben. Gyr. fusif. und alle Windungen an der unteren und äusseren Oberfläehe des Lappens sind gänzlich unverändert.

Schnitt 5, $38 \mathrm{~mm}$ vor der Hinterhaupts-Spitze. Der Cuneus ist nach innen zu vom Präcuneus überlagert, sonst zeigt der Schnitt dieselben Theile wie der vorige, auch den braunen Erweichungs-Heerd an der gleichen Stelle: die Spitze desselben liegt unmittelbar über dem Hinterhorn; von da aus steigen die zwei Grenzlinien divergirend auf, die laterale, welche wieder einen durchgebenden Spalt bildet, durch das Hemisphären-Mark ausserhalb der Fiss. calcarina bis zur Basis des Cuneus, die mediale, längs welcher gesundes und krankes Gewebe noch zusammenhängt, durch den Gyr. ling. durch; nur ist die Divergenz beider Linien etwas geringer, als früher, derart, dass die intacte, untere Hälfte des G. ling. grösser als trüher ist. Somit hat die Erweichung wiederum die Wand der Fiss. calcarina in ibrer ganzen Ausdebnung getrofien. Fiss. coliateralis, G. fusif. und alle übrigen Theile des Schnittes sind unverändert.

Schnitt 6, $44 \mathrm{~mm}$ von der Hinterhaupts-Spitze. Die mediale Seite oberhalb der Fiss. calcarina wird vom Präcuneus eingenommen; zwischen demselben und dem Gyr. ling. schneidet die Oberfläche tief ein, bis auf den Grund des Einschnittes mit Pia mater überzogen; der tiefste Punkt des letzteren liegt nur $4 \mathrm{~mm}$ rom Hinterhorn entfernt, und das gesammte zwischenliegende Gewebe sinkt stark unter das Niveau zurück, ist ganz schlaff, zerklüftet und braun, gleicht also dem des bisher verfolgten Erweichungs-Heerdes, stellt anch die continuirliche Fortsetzung desselben dar. Ferner aber schliesst sich hier an den schlaffen Eeerd nach oben zu eine Erweichungs-Höhle an, welche die Marksubstanz des Präcuueus, und zwar des unteren, jenen Einschnitt überlagernden Theiles desselben und das angrenzende Hemisphären-Mark einnimmt. In's Bereich des braunen Heerdes fällt wiederum die ganze Fiss, calcarina und die Fiss. parietooccipitalis, beide nicht von einander getrennt, da der sie scheidende Cuneus-Stiel in dem zusammengesunkenen Gewebe nicht nachzuweisen ist. Die untere, mediale Grenze des ganzen Heerdes zieht wiederum rom 
inneren Umfang des Hinterhorns durch den Gyr. ling. zur Oberfläche, so dass der obere, die Fiss. calcar. begrenzende Theil des letzteren in die Zerstörung einbegriffen ișt.

Schnitt 7, $52 \mathrm{~mm}$ vor der Hinterhaupts-Spitze. Zwiscben Präcuneus und Gyr. ling. schneidet die Furche, das gemeinsame Verlaufsstück der Fiss. calcar. und Fiss. parieto-occip, als enger Spalt in der Richtung von innen nach aussen $12 \mathrm{~mm}$ weit ein. Die auskleidende Rinde ist ganz schmal und am Dach und Boden der Furche durch einen ErweichungsSpalt fast vollkommen aus der Ungebung ausgeschält. Nur an dem vom Präcuneus gebildeten Dach bängt in Eingangs-Theil der Furche Rinde und Mark fest zusammen, hier ist auch die erstere nicht verschmälert und von normaler Farbe. Von da ab aber ist die abgelöste Rinde nur etwa $\frac{1}{2} \mathrm{~mm}$ dick, gelblich und sohr schlaff; an dem vom Gyr. ling. gebildeten Boden der Windung Jäuft der subcorticale Spalt bis zur medialen Oberfläche durch. Die Rinde ist hier bis $1 \mathrm{~mm}$ dick, gelb, schlaff; beide Spalten stossen in spitzem Winkel auf einander hart über dem Hinterhorn, dessen Durchschnitt ein Dreieck mit nach innen gerichteter Spitze darstellt, und das Ependym des Daches ist durch den unteren Spalt abgelöst. Im unteren Abschnitt des Praecuneus findet sich eine spaltförmige ErweichungsHöhle im Mark, welche von dem die Fiss. calc. umkreisenden Spalt durch eine $1 \mathrm{~mm}$ breite Brücke weisser Substanz geschieden ist.

Schnitt 8, $58 \mathrm{~mm}$ nach vorn von der Spitze des Occipital-Lappens. Die Fiss. calcarina schneidet nur noch $5 \mathrm{~mm}$ tief ein. Die ganze Zerstörung beschränkt sich auf einen Spalt, welcher die Rinde der ganzen Fissur an Dach und Boden umkreist und vollständig von der Marksubstanz ablöst. Der Heerd im Mark des Praecuneus ist verschwunden.

Schnitt 9, $65 \mathrm{~mm}$ vor der Hinterhaupts-Spitze. Gyr. lingualis und Fiss. calcar. sind nicht mehr vorhanden. Ueber dem Gyr. fusif. liegt der Gyr. hippocampi, an dessen medialem Rand eine schmale und oberflächliche gelbe Färbung der Rinde vorhanden ist. Sonst sind alle Theile unverändert.

Auf den übrigen durch die Hemisphäre gelegten Frontalschnitten finden sich keinerlei Erkrankungs-Heerde mehr vor, speciell sind die Stammganglien unverändert.

Die genauere Untersuchung der linken Grosshirnhemisphäre an Frontalschnitten hat zunächst die Bestätigung des früher am unzerlegten Gehirn constatirten Verhältnisses geliefert, dass der Erweichungs-Process sich auf die mediale untere Fläche des Occipital- und angrenzenden Theiles des Temporal-Lappens beschränkt, und̉ zwar vou den an die Oberfläche anstossenden Theilen den Cuneus, Gyr. lingualis und Gyr. fusiformis betrifft; die untere Windung des Cuneus, welche den grösseren Theil seiner medialen Oberfläche liefert, ist mit 
Ausnahme eines circumscripten Punktes der Rinde (Schnitt 1) in ganzer Ausdehnung bis zur Basis zerstört, die obere, von der nur der Kamm an der medialen Fläche zu Tage liegt, besitzt an diesem zwar eine intacte Rinde, aber ein erweichtes Markstratum, sodass der gesammte Cuneus für functionsunfähig gelten darf. Auch der Cuneus-Stiel ist zerstört und abgeflacht und infolge dessen kurz, sodass die Trennung der Fiss. calcarina und Fiss. parieto-occip. weniger weit nach vorn reicht, ihr gemeinsames Verlaufsstück schon weiter hinten beginnt; vom Prae-. cuneus nimmt ein Streifen der medialen Oberfläche, welcher an der Fiss. parieto-occip. entlang zieht, an der Erweichung Theil, aber sein übriger Abschnitt ist unverändert. Der abwärts steigende Theil des Gyr. fornicatus, welcher ihn nach vorn zu in der Begrenzung des gemeinsamen Verlaufsstückes der Furchen ablöst und das Dach desselben bildet, enthält nur auf einem Schnitt (i) kleine Erweichungs-Heerde im Mark, und in derselben Region kommt ein etwas grösserer Heerd im Mark unter dem Praecuneus hinzu. Schliesslich trägt der Gyr. hippocampi an dem medialen Rand eine streifenförmige, Rinde und Mark betreffende ErweichungsZone. Der Gyr. ling., welcher unter der Fiss. calcarina entlang zieht, und der Gyr. fusiformis sind in ihrem an die Oberfläche anstossenden Theil fast in ganzer Längen-Ausdehnung völlig zerstört, bis aut zwei in Schnitt 3 gelegene circumscripte Inseln der Rinde: nehmlich eine kleine intacte Stelle an dem den Eingang der Fiss. calcarina begrenzenden Rand des Gyr. lingualis, und eine am unteren lateralen Rand des Gyr. fusiformis; indessen ist an beiden Stellen das unterliegende Mark erweicht. Die Abplattung der beiden genannten Windungen tritt auf den Frontalschnitten darin klar za Tage, dass die sie trennende CollateralFurche nur in der Spitze des Occipital-Lappens vorhanden, nach vorn hin völlig verstrichen, und ihre Stelle nur durch die Arterie bezeichnet ist, welche im hinteren Theil in die Furche versenkt, vorn an der Oberfläche liegt. Die Erweichung reicht vom Kamm der Windungen durch ihre Rinde und Marksubstanz über ihre Basis hinaus ins Hemisphären-Mark. An beiden Windungen nimmt der Grad und die Ausdehnung der Erweichung von hinten nach vorn zu: die Substanz des Gyr. fusif. ist in Schnitt 6 bis 8 völlig geschwunden, das Ependym des ausgebuchteten 
Hinterhorns liegt unmittelbar unter den weichen Häuten. Offenbar entspricht dies Gebiet der früher bei Eröffnung der Seitenventrikel wahrgenommenen Transparenz des Hinterhorns. Am Gyr. ling. macht sich die Zunahme der Erweichung nach vorn $z u$ in dem Verbalten seines die Fiss. calcarina begrenzenden A bschnittes bemerkbar: Die Fiss. calc. besitzt nur auf der ersten Schnittfläche (10 $\mathrm{mm}$ vor der Hinterhaupt-Spitze) und auf den von dieser nach hinten folgenden Mikrotomschnitten die gewöhnliche Form der sich in der Tiefe flächenhaft ausbreitenden Furche mit Dach, Boden und Grund, derart, dass die Furche selbst schräg von oben-innen nach unten-aussen, der Grund ungefähr vertical steht; letzterer breitet sich sowohl nach unten, als nach oben von der Furche aus, wird also vom Cuneus und vom Gyr. ling. überwölbt. Weiter nach vorn wird in Folge des Zusammensinkens der betheiligten Windung der Verlauf der Furche in der Art verzogen, dass dieselbe nur einen von oben nach unten einschneidenden Spalt mit medialer und lateraler Wand darstellt; die laterale begreift Grund und Dach in sich, weil der eingesunkene Cuneus sich nicht mehr überwölbt, die mediale, vom Gyr. ling. gebildete den ursprünglichen Boden der Furche; die Grenze zwischen Grund und Dach wird nur durch eine in der Furche laufende Arterie bezeichnet. Erst weiter vorn, wo der intacte und normal gewölbte Gyr. fornicatus das Dach der Fiss. calcar., bezw, der vereinigten Fiss. calc. und parieto-occipitalis bildet, rückt der Eingaug der Furche wieder nach abwärts, sodass dieselbe wieder einen mehr horizontalen Verlauf bekommt. Von der Wandung der Fiss. calcarina ist trotz der fast totalen Zerstörung der medialen Oberfläche in Schnitt. 1 u. 2 , also bis $z$ u $18 \mathrm{~mm}$ vor der Hinterhaupt-Spitze, ein beträchtlicher Theil der Rinden- und Marksubstanz völlig intact, von da an nach vorn aber die gesammte Wand zerstört, und zwar am frunde der Furche bis weit in das Hemisphären-Mark hinein, ebenso die gesammte Wand im hinteren Abschnitt des gemeinsamen Verlaufsstücks der beiden Furchen, während im vordersten Abschnitt des letzteren (Schnitt 6 u. 7) das vom Gyr. fornicatus gebildete Dach wieder normal, oder wenigstens nur von kleiuen Markheerdchen durchsetzt ist, 
bei völliger Zerstörung des Grundes und der medialen Wand. Der erhaltene Theil im hinteren Abschnitt betrifft auf Schnitt 1 den gesammten breiten Grund, an dem die Rinde und die dem Hemisphärenmark angehörige weisse Substanz völlig unverändert sind, und den dem Grund gegenüberstehenden Abschnitt des Bodens (Gyr. lingualis), an welchem die Rinde und ein allerdings schmaler Streifen der Marksubstanz normale Structur besitzt; dagegen ist der nach dem Eingang zu liegende 'Theil des Bodens und das ganze vom Cuneus gebildete Dach in der Rinde von Heerdchen durchsetzt, im Mark continuirlich erweicht, und somit sicherlich als functionsunfähig anzusehen. In Schnitt 2 ist vom Eingang der gerade nach abwärts laufenden Fissur die laterale Wand (Cuneus) total zerstört, während der tiefere Theil der Furche eine völlig intacte Rinde an medialer und lateraler Seite besitzt, welche von normaler Marksubstanz umgeben ist, und zwar nach aussen und nach unten von dem in ganzer Breite unveränderten Hemisphären-Marke, nach innen nur in einer $1 \mathrm{~mm}$ breiten Schicht des Markstratum des Gyr. ling., und an der medialen Seite zieht sich dieser Streifen normaler, weisser Substanz mit unveränderter Rinden-Bekleidung bis zum Eingang der Fissur. Also völlig unberührt von dem Erweichungs-Process ist die Wand der Fissur am grösseren Theil des Grundes und der unteren Umbiegungsstelle, als zweifelhaft bezüglich der Functionsfähigkeit, weil nur noch mit einer subcorticalen Schicht von Mark versehen, am Boden der Fissur, und als völlig unbrauchbar am Dach. Alle übrigen Theile des Hinterhaupt- und Schläfenlappens erwiesen sich als durchaus normal.

An der rechten Hemisphäre liegen die Verhältnisse weit einfacher: die Erkrankung folgt hier fast ohne Abweichung der Fiss. calcarina vom hintersten bis zum vordersten Ende, wobei die Tiefe der Zerstörung zunächst (Schnitt 1-6) nur geringe Schwankungen erfährt, um sich schliesslich vorn auf eine subcortikale Zone der Wand zu beschränken. Hierzu kommt nur eine Aushöhlung des untersten Praecuneus-Abschnittes durch Erweichung seiner Marksubstanz. Sämmtliche anderen Abschnitte des Occipital- und Temporallappens sind unbetheiligt. Bis zu $44 \mathrm{~mm}$ vor der hintersten Spitze (Schnitt 6) umfasst die Zer- 
störung die gesammte Wand der Fiss. calcarina; betheiligt sind dabei der obere Theil des Gyr. ling. als Boden der Furche, die untere Cuneus-Windung als Dach, und der flächenhafte Grund mit dem Hemisphären-Mark. Nach allen Seiten hält sich die Erweichungs-Grenze in annähernd gleicher Entfernung von der Fissur, sodass die letztere die Axe des Erweichungs-Keiles bildet; überall aber trifft die Erkrankung nicht nur die Rinde der Furche, sondern greift weit in die zugehörige Marksubstanz ein. Der ganze Heerd ist in Folge der Bildung von Spalten an seiner Grenze fast ganz aus dem benachbarten Parenchym herauszulösen. Auf der medialen Oberfläche ist die Erweichung am Gyr. ling. nur als schmaler Streifen, am Cuneus als breitere Zone sichtbar. Von Schnitt $7(52 \mathrm{~mm})$ an zieht sich die Zerstörung ganz auf die Auskleidung der Furche, der vereinigten Fiss. calearina und Fiss. parieto-occip. zurück, die mediale Oberfläche bleibt unbetheiligt; und zwar ist von hier an die Rinde der Furche durch einen Spalt völlig ausgelöst bis auf einen kleinsten circumscripten Bezirk des Daches am Eingang auf Schnitt 7; mit dem vorderen Ende des Gyr. ling. und der Fiss. calcarina endet auch die Erweichung.

Seitdem Gratiolet die nach ihm benannten Faserzüge entdeckt und von den primären Opticus-Centren, dem Corpus geniculatum externum, dem oberflächlichen Mark der vorderen Vierhügel und den äussersten Schichten des Thalamus opticns in gesonderten Bündeln rückwärts ziehen und sich in der Rinde der Hinterhaupts-Lappen verlieren sah, hat man eine Beziehung dieser Hirnrindentheile zu den Gesichtswahrnehmungen wohl vermuthen müssen. Diese Vermuthung erhielt eine kräftige Stütze in den bekannten Untersuchungen von H. Munk, welcher durch Versuche an Hunden und Affen bei diesen Thieren eine Sehsphäre in der Rinde des Occipital-Lappens nachgewiesen hat. Seitdem hat man in ihr das eigentliche Centrum der Sehfunction angenommen. Vielfache Sektions-Befunde beim Menschen haben ergeben, dass Zerstörungen des Hinterhaupt-Lappens zur homonymen Hemianopsie der entgegengesetzten Seite führen. Nothnagel schreibt bereits in seiner im Jahre 1879 erschienenen topischen Diagnostik der Gehirnkrankheiten S. 587: „Wenn die 
homonyme Hemianopsie plötzlich unter dem Bilde eines apoplectischen Insultes auftritt, der ophthalmoskopische Befund negativ ist, und keine weiteren Erscheinungen daneben bestehen, die Hemianopsie demnach die einzige pathologische Veränderung ist, so wird der Heerd im Occipital-Lappen zu suchen sein ", und belegt diesen Satz durch eigene und fremde Beobachtungen.

Welche Theile des Occipital-Lappens aber als Sehcentrum anzusprechen sind, blieb noch lange unbekannt. Noch Mauthner, der in seiner bekannten, 1881 erschienenen Schrift über „Gehirn und Auge" eine grosse Zahl von Laesionen der Hinterhaupts-Lappen, die anatomisch festgestellt worden waren, gesammelt hat, konnte eine genauere Localisirung nicht versuchen, und selbst Förster hat im Jahre 1890 in seiner oben-erwähnten Arbeit über Rindenblindheit nur auf die Rinde des Occipital-Lappens ohne genauere Begrenzung hinweisen können. Erst Henschen ist es gelungen, die Sehsphäre des Menschen genauer zu localisiren. Dieser Forscher, dessen wahrhaft monumentales Werk „Klinische und anatomische Beiträge zur Pathologie des Gehirns", 3 Foliobände, Upsala 1890-1896, nicht genug bekannt and gewürdigt worden ist, hat das Verdienst, auf Grund einer grossen Anzahl eigener und aus der Literatur gesammelter, klinisch und anatomisch sorgfältig untersuchter und kritisch analysirter Fälle gezeigt zu haben, dass es die Fissura calcarina und die ihr zunächst benachbarten Rindentheile der Medianfläche sind, welche als die centralen Endigungen der Sehfasern des Nervus opticas angesehen werden müssen. Er gelangte zu diesem Schlusse, indem er einerseits Fälle von Erweichungen der Rinde an der lateralen Fläche, und solche von Zerstörungen der ventralen Fläche sammelte, welche ohne Hemianopsie verlaufen waren, und ihnen andererseits eine grosse $\mathrm{Zahl}$ von Fällen gegenüberstellte, welche Läsionen der Fissura calcarina darboten, und in denen die Erscheinungen der Hemianopsie deutlich nachgewiesen waren. Er konnte sogar Beobachtungen mittheilen von Läsionen der medialen Flächen in ihrem oberen Theile (Cuneus), welche ohne Einfluss auf die Sehfunction geblieben waren.

Die seit Henschen's Veröffentlichungen bekannt gewordenen, zur Autopsie gelangten Fälle haben jenen wichtigen Satz im Wesentlichen durchaus bestätigt: Namentlich sind die von 
Vialet und Déjérine veröffentlichten Beobachtungen von reiner, einseitiger homonymer Hemianopsie, bei denen als einzige Läsion eine Erweichung in der Fissura calcarina und ihrer Umgebung gefunden wurde, geeignet, den obigen Satz zu stützen. Auch die anatomische Untersuchung des Förster'schen Falles von Sachs and die unseres Falles, welche beide eine doppelseitige Hemianopsie betrafen, stehen mit der Henschen'schen Annahme durchaus im Einklang. Henschen ist aber noch weiter gegangen und hat eine nähere Localisirung versucht. Aus einem Falle von Wilbrand (No. 149 Bd. II S. 327) and zwei Fällen von Reinhard (Fall 148 und 152) schliesst er mit einer gewissen Wahrscheinlichkeit, dass das Rindenfeld der Macula lutea in den vorderen Theilen der Fissura calcarina gelegen ist; er giebt aber selbst zu, dass die Reinhard'schen Fälle nicht beweiskräftig sind, weil sie demente Patienten betrafen, bei denen eine genaue Gesichtsfeld-Aufnahme kaum möglich war, und dass ,in der Literatur bisher kein unzweideutiger Fall existirt, welcher diese Frage zur endgültigen Lösung bringen könnte".

Es scheint uns nun, dass unser Fall, im Verein mit dem Förster'schen von Sachs anatomisch untersuchten Fall, geeignet ist, diese von Henschen angegebene Lücke auszufüllen. Wir fanden auf beiden Augen in einem minimalen Gesichtsfelde eine vortreffliche centrale Sehschärfe, welche die volle Functionsfähigkeit der Fasern der Fovea centralis bewies. Im rechten Occipital-Lappen fand sich die ganze Region der Fissura calcarina und derjenigen Theile, welche als Sehcentrum in Betracht kommen können, zerstört. Im linken Occipital-Lappen war die Erweichung noch viel umfangreicher. Hier schien ausser der ganzen Fissura calcarina der ganze Cunens, sowie der ganze Gyrus lingualis und der Gyrus fusiformis zerstört, aber im Grunde der Fissura calcarina zeigte sich ungefähr in einer Strecke, welche von der Nähe der Hinterhaupts-Spitze bis za einem $18 \mathrm{~mm}$ vor ihr gelegenen Punkte reichte, eine grössere Partie der Rindenund Marksubstanz völlig intact. Dieser gesund gebliebene Theil hat eine nicht unbedeutende Flächen-Ausdehnung; denn es muss berücksichtigt werden, dass die Fissur in dieser Gegend nicht einen einfachen Spalt darstellt, sondern dass ihr Boden, wie die 
Figuren L. 1 und L. 2 zeigen, den über ihm liegenden Cuneus und den unter ihm liegenden Gyrus lingualis gegen $5 \mathrm{~mm}$ weit unterminirt und entfaltet wohl einen Flächenraum von vielleicht $2 \mathrm{qcm}=200 \mathrm{qmm}$ einnehmen würde. Da die Macula lutea im Auge nur eine Fläche von $3 \mathrm{qmm}$ bedeckt, so sieht man hieraus, dass das ihr zugehörende Rindenfeld eine mindestens 60 mal grössere Flächen-Ausdehnung besitzt $^{1}$ ). Der Befund lässt keine andere Deutung zu, als die, dass an dieser Stelle die Fasern des centralen Theils der Macula lutea ihre Endigungen fanden. Zugleich ergiebt sich mit Notbwendigkeit, dass diese Partie der Hirnrinde die Macula lutea beider Augen versorgt.

In vollkommener Uebereinstimmung mit unserem Befunde hat Sachs') in dem Foerster'schen Falle, in welchem zuerst der linksseitige und erst nach vier Jahren der rechtsseitige Heerd aufgetreten war, in dem zuletzt entstandenen Heerde einen schmalen Streifen intacter Substanz im hintersten Abschnitt des Bodens der Fissura calcarina gefunden, welche allem Anscheine nach mit Balken- und Stabkranzfaserung im Zusammenhang geblieben war, und auf welchen er die Erhaltung des kleinen centralen Gesichtsfeldes beider Augen zurückführte.

Die relativ grosse Ausdehnung, welche das Macula-Rindenfeld demnach in beiden Hemisphären besitzt, ist der anatomische Ausdruck für die grosse Dignität, welche der Macula lutea als der Vermittlerin des centralen Sehens beim Menschen zukommt. $\mathrm{Da}$ in unserem Falle dieses intacte Rindenfeld in der linken Hemisphäre, in dem Sachs'schen Falle in der rechten Hemisphäre gefunden wurde, so folgt hieraus, wenn es dafür noch eines besonderen Beweises bedürfte, dass in jedem der beiden

1) Es liegt nahe, diese grosse Ausdehnung des Rindenfeldes in Beziehung zu setzen mit der grossen Zahl der Associationen, welche zwischen dem centralen Sehen einerseits und zahlreichen psychischen und anderen Nerven-Functionen andererseits bestehen. Wenn der blosse Anblick eines Gegenstandes das eine Mal Frende, das andere Mal Furcht, Sebrecken oder Scham erregen kann, ein drittes Mal einen Brechreiz auslöst, so müssen für alle diese Wirkungen Associationsfasern vorhanden sein, die das Macula-Centrum mit anderen Centren verbinden, und diese Fasern beansprachen einen grossen Raum.

2) Arbeiten aus der psychiatrischen Klinik in Breslau. Herausgegeben von C. Wernicke, Heft II S. 78. Leipzig 1895. 
Hinterhaupts-Lappen ein solches Macula-Centrum vorhanden is t.

Gegen die aus unsern positiven Befunden gefolgerte Localisirung des Macula-Centrums im hintersten Theile der Fissura calcarina scheint der Fall von Küstermann ${ }^{1}$ ) zu sprechen, welcher nach doppelseitiger Hemianopsie bei einem 51 jäbrigen Potator, welcher vielleicht an Alkoholvergiftung zu Grunde ging, auf beiden Augen einen nach links und oben gelegenen Rest des Gesichtsfeldes erbalten sah. In diesem Falle zeigte die Section, dass in beiden Hirnhemisphären ein kleines Stück des Bodens der Fissura calcarina, ca. $3 / 4 \mathrm{~cm}$ vor der Spitze des HinterhauptLappens gelegen, intact geblieben war. Auf diesen nahe dem hintern Ende der Fissur gelegenen, unzerstörten Theil bezieht Küstermann die Erhaltung des nach links oben gelegenen Gesichtfeld-Restes, und verlegt darum das Macula-Centrum in den vordern Theil der Fissur. Der Fall scheint uns jedoch nicht beweisend zu sein. Die doppelseitige Hemianopsie konnte erst in den letzten 7 Tagen vor dem tödtlichen Ausgange beobachtet werden, und es dürfte nicht leicht gewesen sein, bei dem elenden, stark dem Trunk ergebenen und an Krampfanfällen leidenden Patienten die Gesichtsfeld-Ausdehnung sicher zu bestimmen. Ausserdem unterscheidet sich der Küstermann'sche Fall auch dadurch von dem unsrigen, dass bei ihm beiderseits symmetrisch ein Stück intacter Rinde gefunden wurde.

Worauf im Sachs'schen Falle und im unsrigen, sowie auch in den übrigen, theils zur Section gelangten Fällen (SchmidtRimpler and Peters) theils in den analogen, nur intra vitam beobachteten Fällen von beiderseitiger homonymer Hemianopsie die Integrität des Macula-Centrums beruht, lässt sich zur Zeit mit Sicherheit noch nicht angeben. Dass die hintere Partie der Fissura calcarina unter besonders günstigen Ernährungsbedingungen steht, kann wenigstens nicht bewiesen werden.

In den meisten genau untersuchten Fällen, auch denen von einseitiger Hemianopsie, hat sich der Gyrus lingualis in grösserem Umfange erweicht gefunden; auch wurde meistens die untere Hälfte des Cuneus zerstört gesehen. Man darf hieraus den Schluss ziehen - und in dieser Beziehung sind gerade die Fälle beweisend, in denen die einseitige homonyme Hemianopsie die

1) Monatssebr. f. Psychiatrie u. Neurologie Jahrg. 1897. S. 335-353. 
einzige klinisch nachweisbare Störung darstellte - dass das optische Rindenfeld doch räumlich weiter ausgedehnt ist, als Henschen angiebt, und mindestens noch einen ansehnlichen Theil des Gyrus lingualis einnimmt. Die Erweichungen von Theilen des Gyrus fusiformis sind zwar ebenfalls wiederholt gefunden worden, doch nicht so constant, dass sie für die Hemianopsie mit verantwortlich gemacht werden müssten. Dagegen waren sie in dem Sachs'schen Falle und in dem unsrigen in dem zuletzt aufgetretenen Heerde, welcher auch die OrientirungsStörungen hervorgebracht hatte, so deatlich ausgesprochen, dass es nahe liegt, sie mit letzteren in Beziehung zu setzen, und in dem Gyrus fusiformis das Centrum für die topographischen Vorstellungen $\mathrm{zu}$ erblicken.

Unser Fall giebt noch zu mancherlei andern Bemerkungen Anlass, welche sich auf die Erhaltung der Pupillenreaction, den Farbensinn und das Ausbleiben jeder mit dem Augenspiegel wahrnehmbaren Atrophie der Papilla nervi optici beziehen. Hier wollen wir nur den letzten Punkt kurz berühren. In dem Foerster'schen Falle waren die Papillen noch nach 4 Jahren, in dem unsrigen noch nach 10 Monaten nach dem zweiten Anfalle völlig normal geblieben. Man kann sich dies dadurch erklären, dass die Fasern der Sehnerven zwei verschiedenen Reihen von Neuronen angehören: einer centralen, welche von der Hirnrinde zu den grossen Stammganglien reicht, und einer peripherischen, welche von den letzteren durch die Tractus und Nervi optici bis zur üussersten Netzhautschicht geht. Da die Neurone als trophische Einheiten angesehen werden und degenerative Processe sich nur innerhalb eines Neurons verbreiten, da ferner der krankbafte Process in den vorliegenden Fällen in den centralen Neuronen vor sich geht, so erklärt sich hieraus, dass der Sehnerv und die Papille auch noch nach langer Zeit gesund befunden wurden. Allein, unübersteiglich scheint die Schranke, welche das Ende des Neurons bildet, doch nicht zu sein. Henschen führt Fälle an, in welchen nach langjährigem Bestehen von hochgradigen Laesionen beider Augäpfel eine auffallende Atrophie in beiden Occipital-Lappen, namentlich in der Rinde der Fissura calcarina, gefunden wurde. Auch bei einem Einäugigen, welcher 41 Jahre nach dem Verlust seines linken Auges gestorben war, giebt er an, in der Fissura calcarina beider Gehirnlappen eine 
Atrophie constatirt zu haben. Sachs hat in dem Försterschen Falle in der linken, seit 8 Jahren erkrankten Gehirnhälfte das Pulvinar des Thalamus fast faserleer und den Tractus opticus ungemein geschrumpft gefunden. Im Tractus konnte er einen von lateralwärts her sich tief in ihn hineinschiebenden Degenerations-Streifen bis in das Chiasma verfolgen. Danach scheint es, als ob die Degeneration nach Zerstörung des optischen Rindenfeldes sich doch über die Stammganglien hinaus nach der Peripherie fortsetzen kann; nur bedarf es zur Ueberschreitung der Neuronen-Grenze vieler Jahre, und gewöhnlich werden die Kranken zu Grunde gehen, ehe die Atrophie das vordere Ende des Sehnerven erreicht hat.

Wir können die Ergebnisse unserer Untersuchuug in folgende Sätze zusammenfassen:

1) Als optisches Rindenfeld sind der ganze Boden der Fissura calcarina, ihre Seitenwände, der untere Theil des Cuneus und ein grosser Theil des Gyrus lingualis zu betrachten.

2) Das Centrum für die Sehfasern der Macula lutea liegt im Boden und in den Seitenwänden des hintersten Abschnittes der Fissura calcarina beider Hemisphären und hat eine relativ sehr grosse Flächen-Ausdehnung.

3) Es ist höchst wahrscheinlich, dass der grösste Theil des Gyrus fusiformis mit dem Orientirungs-Vermögen in nahem $\mathrm{Zu}$ sammenhange steht.

$$
\text { Erklärung der Tafeln. }
$$

Taf. XII. Fig. 1 stelit die Ausdehnung der Erweichang der medialen und unteren Fläche des Occipital- und Temporallappens der linken Hemisphäre dar. Die erweichten Windungen sind, um den Gefässverlauf zu zeigen, ein wenig auseinander gezogen. Der Doppelpfeil bezeichnet die Stelle des Thrombus.

Fig. 2 zeigt die mediale und untere Fläche der hinteren Bälfte der recht. Hemisphäre mit dem weniger ausgedebnten Erkrankungs-Heerde.

Taf. XIII. L 1-6 Frontalschnitte der linken Hemispbäre:

L 1: Schnitt 1, $10 \mathrm{~mm}$ vor der Hinterhaupt-Spitze,
$\mathrm{L} 2$ :
2,18
L 3 :
3,24
$\mathrm{L} 4$ :
4,3
I. 6:
6,45
$" \quad "$
" " "

R 2 und 3 Frontalschnitte der recbten Hemisphäre:

R 2: Schnitt 2; $18 \mathrm{~mm}$ vor der Hinterhaupt-Spitze,

R 3

$$
3,24
$$




$\begin{array}{ll}\text { Cun. 1: } & \text { Erste Cuneus-Windung; } \\ \text { Cun. 2: } & \text { Zweite Cuneus-Windung; } \\ \text { Cs : } & \text { Cuneus-Stiel; } \\ \text { Pc: } & \text { Praecuneus; } \\ \text { G. ling.: } & \text { Gyr. lingualis; } \\ \text { G. fus.: } & \text { Gyr. fusiformis; } \\ \text { G. forn.: } & \text { Gyr. fornicatus; } \\ \text { F. cun.: } & \text { Fiss. cunei; } \\ \text { F. calc.: } & \text { Fiss. calcarina; } \\ \text { F. coll.: } & \text { Fiss. collateralis; } \\ \text { F. call.-mar.: } & \text { Fiss. calloso-marginalis; } \\ \text { F. par.-occ.: } & \text { Fiss. parieto-occipitalis; } \\ \text { Art. c.: } & \text { Arteria collateralis; } \\ \text { H: } & \text { Hinterborn. }\end{array}$

\title{
XXIII.
}

\section{Die Schilddrüse als entgiftendes Organ}

\author{
von \\ F. Blum, \\ Frankfurt a. M.
}

(Nach einem in der Sitzung des Vereins für innere Medicin zu Berlin am 12. Juni 1899 gehaltenen Vortrage.)

Vor dem Beginne der 80 er Jahre konnte man kaum von einer Physiologie der Schilddrüse sprechen; in dem gänzlich unerforschten, geheimnissvollen Gebiete der Blutgefäss-Drüsen lag die Thyreoidea neben Milz, Thymus, Nebenniere und Hypophyse still begraben. Wenn auch Schiff schon im Jahre 1859 die Beobachtung gemacht hatte ${ }^{2}$ ), dass die Wegnahme der Schilddrüse bei Thieren schwere Störungen hervorrufen könne, und obgleich der Württemberger Arzt Si ck ${ }^{2}$ ) 1867 bereits die Kranken-

1) M. Schiff, Untersuchungen über die Zuckerbildung in der Leber und den Einfluss des Nervensystems auf die Erzeugung des Diabetes. Würzburg, 1859 , S. $61 \mathrm{f}$.

2) P. Sick, Ueber die totale Exstirpation einer kropfig entarteten Schilddrüse, und über die Rückwirkung dieser Operation anf die CirculationsVerhältnisse im Kopf. Württemb. Med. Correspondenzblatt 1867, No. 25. 